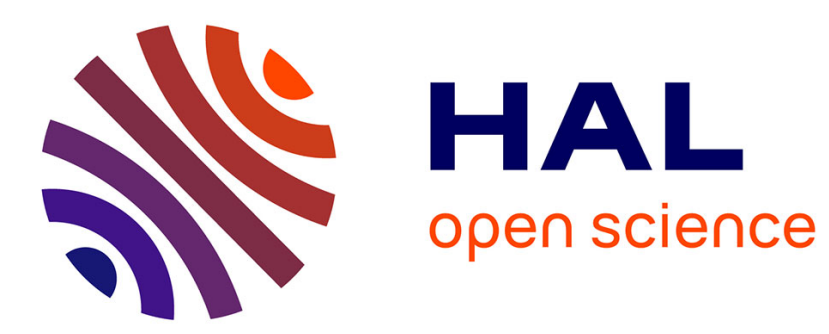

\title{
Correlation among tardiness-based measures for scheduling using priority dispatching rules
}

\author{
Nasser Mebarki, Atif Muhammad Shahzad
}

\section{To cite this version:}

Nasser Mebarki, Atif Muhammad Shahzad. Correlation among tardiness-based measures for scheduling using priority dispatching rules. International Journal of Production Research, 2013, 51 (12), pp.3688 - 3697. 10.1080/00207543.2012.762131 . hal-01740868

\section{HAL Id: hal-01740868 \\ https://hal.science/hal-01740868}

Submitted on 22 Mar 2018

HAL is a multi-disciplinary open access archive for the deposit and dissemination of scientific research documents, whether they are published or not. The documents may come from teaching and research institutions in France or abroad, or from public or private research centers.
L'archive ouverte pluridisciplinaire HAL, est destinée au dépôt et à la diffusion de documents scientifiques de niveau recherche, publiés ou non, émanant des établissements d'enseignement et de recherche français ou étrangers, des laboratoires publics ou privés. 


\title{
ARTICLE
}

\section{Correlation Among Tardiness Based Measures for Scheduling using Priority Dispatching Rules}

\author{
Nasser MEBARKI ${ }^{a *}$ and Atif SHAHZAD ${ }^{b}$ \\ ${ }^{a, b}$ Institut de Recherche en Communications et Cyberntique de Nantes, \\ 1 rue de la Noe BP 92101, 44321 Nantes, France.
}

( $v 1.0$ released Apr 2012)

One of the most used methods to schedule manufacturing systems is to use priority dispatching rules (pdrs). It is frequently desired to distinguish the behavior of pdrs in regards with tardiness based performance measures. But, the relation among these performance measures is generally not obvious even for the simple scheduling strategies such as pdrs. In this paper, we first focus on the maximum tardiness that is a very interesting performance measure for the decision-maker in the shop. However, because of its singularity, it is not trivial to assess it. We conducted a simulation study on a benchmark model of a dynamic job-shop system to evaluate the relative performance of a set of pdrs chosen either because these are extensively used or because these exhibit very good performance. Based upon the distribution of the maximum tardiness of these benchmark pdrs, we identify two sub-sets of pdrs. From this, we conducted experiments on the root-mean-square tardiness, which is used to distinguish a system with a few highly tardy jobs from a system with a lot of little tardy jobs. The experiments made show a positive correlation between maximum tardiness and root-mean-square tardiness. Because of the fact that the root-mean-square tardiness is an aggregate measure, it is much more easier to assess it than the maximum tardiness. This provides an opportunity to predict the relative performance of pdrs in regards with the maximum tardiness as well as the width of the tardiness by evaluating root-mean-square tardiness only.

Keywords: Scheduling; Tardiness; Simulation; Priority Dispatching; Stochastic Processes

${ }^{*}$ Corresponding author. Email: nasser.mebarki@univ-nantes.fr

ISSN: $1478-6451$ print/ISSN 1478-646X online

(C) 2012 Taylor \& Francis

DOI: $10.1080 / 1478645 Y Y x x x x x x x$

http://www.informaworld.com 


\section{Introduction}

Priority dispatching rules are widely used to dynamically schedule the operations in a shop. However, their efficiency depends on the performance criteria of interest (Dominic et al. 2004). Tardiness is an important criteria in real systems which can be measured through several performance measures. These measures are used to rank different scheduling strategies under different conditions. However, the superiority of one strategy over the other is not obvious in general, for a mix of performance measures and conditions (Mattfeld and Bierwirth 2004).

A priority dispatching rule determines a value of priority index, $\kappa_{j}$, for any job $j$ in a given scheduling situation, allowing a procedure to use these priority indices for selecting one of the schedulable jobs waiting to be started next on an idle machine (Baker 1974, Panwalker and Iskander 1977). For identifying the characteristics and making a comparative study of the behavior of different priority dispatching rules, it is important to classify and establish a relative ranking of the priority dispatching rules especially in regards with the performance measures for which these are intended to be used.

In this paper we are only interested in tardiness based performance and especially with the maximum tardiness which gives the worst-case performance regarding the tardiness and thus can be of great interest for the decision-maker in the shop. We present an analysis of tardiness based performance measures for a set of frequently used priority dispatching rules is made using simulation in order to highlight the similarities/dissimilarities in the behavior of these priority dispatching rule. The worst-case behavior in regards with tardiness is very difficult to identify due to its value at a single-point only. However, it is possible to establish some guidelines in predicting this worst-case behavior. For this, we use the properties of root-mean-square tardiness to identify two different sets of rules.

The rest of the paper is organized as follows. We will first discuss different tardiness based performance measures frequently used in literature on job shop scheduling problem. This follows with a description of experimental setup and the simulation procedure used to evaluate the performances of the priority dispatching rules chosen in regards with tardiness based performance measures. In Section 4, results showing the correlation among two very important measures, maximum tardiness $\left(T_{\max }\right)$ and root-mean-square tardiness $\left(T_{r m s}\right)$ are presented along-with discussion on these results. Finally, some conclusions are drawn which are presented in the last section.

\section{Tardiness in Dynamic Scheduling}

Dynamic scheduling of manufacturing systems for due-date based objectives has received considerable attention from practitioners and researchers due to the importance of meeting due-dates in most industries (Raman et al. 1989, Mainieri and Ronconi 2010). Unfortunately, the study of due-date based objectives is much more complicated than

the flow-time based objectives (Baker and Bertrand 1982). To what extent the goal of keeping the due-dates is achieved, is judged by tardiness based measures (French 1982). However, there is no single universally accepted measure on this dimension.

The tardiness of a job is computed as $T_{j}=\max \left(0, C_{j}-d_{j}\right)$ with $C_{j}$ as the completion date and $d_{j}$ is the due-date of the job $j$. It causes potential losses in terms of bad reputation, higher stock-holdings, rush shipping costs and possibly contractual penalties. To measure the effective performance of the system in regards with the tardiness, there are several performance measures of interest. Generally it is not sufficient to assess the 
performance of a schedule with a single tardiness based performance measure alone (Laslo et al. 2008, Laslo 2010). In the following subsection, the mostly used tardiness based performance measures are described. This follows with a derivation of a relation between two very important tardiness based measures, i.e. $T_{\max }$ and $T_{r m s}$, for stochastic models.

\subsection{Tardiness Based Measures}

The most used performance measure to evaluate the tardiness is the total tardiness (i.e., $\Sigma_{j} T_{j}$ ). While using simulation to compare two different scheduling strategies, the number of completed jobs is usually fixed. Thus, in that case, the mean tardiness (i.e., $\bar{T}$ ) is equivalent to the total tardiness. This measure represents an average level of customer satisfaction in terms of delivery performance (Lejmi and Sabuncuoglu 2002).

The number of tardy jobs $\left(\Sigma U_{j}\right)$ or an equivalent measure namely percentage tardiness $\left(\% T=\Sigma U_{j} / n \times 100\right)$, is also extensively used in literature on comparative study of pdrs. However this measure is risky as standalone measure due to its discrete nature and resulting instability (Kemppainen 2005).

The conditional mean tardiness (i.e., $C M T=\frac{\Sigma_{j} T_{j}}{\Sigma U_{j}}$ ) measures the average amount of tardiness for the completed jobs which are found to be tardy. This performance measure is, however, not regular. It means that it can decrease while the completion times are not decreasing (French 1982).

For the judgment of relative performance of pdrs in regards with tardiness, the shape of the tardiness distribution must be considered. Overall performance of the system will be disturbed heavily due to a very large delay for a job in comparison with a small constant (or varying very little) tardiness values among several jobs (Moser and Engell 1992). In this case, the maximum tardiness can be of great interest for the decision-maker in the shop. It is defined as

$$
T_{\max }=\max _{j} T_{j}
$$

It is an indication of a worst-case behavior of a rule during a particular experiment. When there are tardy jobs, the maximum tardiness is equivalent to the maximum lateness. The lateness of a job $j, L_{j}=C_{j}-d_{j}$ which is positive when job $j$ is completed late and negative when it is completed early (Pinedo 2008). Chang and Su (2001) state that "Minimizing maximum lateness $\left(L_{\max }\right)$ is important if all preceding activities must be completed before the rest can begin in a project". Moreover, (Malve and Uzsoy 2007) show that this performance measure can be of great interest for the semiconductor industry "since this will avoid making some jobs early at the expense of others being extremely late-it tends to spread the deviations evenly across all jobs". This objective can also be measured by the root-mean-square tardiness, defined as

$$
T_{r m s}=\sqrt{\frac{1}{n} \Sigma_{j} T_{j}^{2}} .
$$

It permits to differentiate a system which presents a little number of tardy jobs with higher tardiness from a system which presents a lot of tardy jobs with low tardiness. This performance measure exhibits higher values for the first kind of systems (Moser and Engell 1992). 


\subsection{Relating $T_{\max }$ and $T_{r m s}$ in stochastic models}

With stochastic models, tardiness $(T)$ being a real-valued random variable, its expectancy is the first central moment and its variance is the second central moment. It is estimated from the results of the simulation model and the estimation is computed as

$$
s_{T}^{2}=\frac{1}{n-1} \sum_{j=1}^{n}\left(T_{j}-\bar{T}\right)^{2},
$$

$n$ being the number of jobs, $\bar{T}$ being the estimation of the expectancy $\left(i \cdot e \cdot \bar{T}=\frac{\Sigma_{j} T_{j}}{n}\right)$. Thus we have

$$
\begin{aligned}
s_{T}^{2} & =\frac{1}{n-1} \sum_{j=1}^{n}\left(T_{j}^{2}+\bar{T}^{2}-2 \bar{T} T_{j}\right) \\
& =\frac{1}{n-1}\left(\sum_{j=1}^{n} T_{j}^{2}+n \bar{T}^{2}-2 \bar{T} \sum_{j=1}^{n} T_{j}\right) \\
& =\frac{1}{n-1}\left(\sum_{j=1}^{n} T_{j}^{2}+n \bar{T}^{2}-2 \bar{T} n \bar{T}\right) \\
& =\frac{1}{n-1}\left(n T_{r m s}^{2}-n \bar{T}^{2}\right) \\
& =\frac{n}{n-1}\left(T_{r m s}^{2}-\bar{T}^{2}\right) .
\end{aligned}
$$

This implies that with the root-mean-square tardiness and mean tardiness, we can compute immediately an unbiased estimate of the variance of the tardiness.

We want to distinguish the behavior of different pdrs in regards with tardiness based performance measures. In this study, we are interested in the maximum tardiness $\left(T_{\max }\right)$ that is of much practical significance for the decision-makers in the shop. In the next section, we first present a set of experiments conducted on a set of pdrs for $T_{\max }$ and $T_{r m s}$ in a job shop scheduling environment.

\section{Experiments}

In order to try to make a comparison as relevant as possible, our sample model is a job-shop model used by several researchers. For example, (Eilon and Cotterill 1968) have used this model to test the effects of the SIx rule, (Baker and Kanet 1983) to demonstrate the benefits of the MOD rule, (Baker 1984) to examine the interaction between dispatching rules and due-dates assignment methods, (Russell et al. 1987) to analyze the effects of the CoverT rule comparing with several other pdrs, and (Schultz 1989) to demonstrate the benefits of the CEXSPT rule, (Pierreval and Mebarki 1997) to evaluate the benefits of their pdrs shifting strategy.

The system is a four machine job-shop. Each machine can perform only one operation at a time. The number of operations of the jobs processed in the system follows a discrete 
uniform distribution between 2 and 6 . The routing of each job is fixed and assigned randomly with limited revisits policy. More precisely, when a job leaves a machine and needs another machine for the execution of subsequent operation, each machine has the same probability to be the next, except the one just released, which cannot be chosen. The processing times of operations on machines follow a negative exponentially distribution with a mean of one.

The arrival of jobs in the system is modeled as a Poisson process and are simulated over long time periods. Since shop utilization, $\eta$ is given as

$$
\eta=\frac{\lambda}{\mu}
$$

where $\lambda$ is the job arrival rate and $\mu$ is the service rate of jobs, given as

$$
\mu=\frac{1}{\bar{p}}=1,(\bar{p} \text { being the average processing time of jobs }),
$$

the shop utilization becomes a function of the job arrival rate. This means that by manipulating the job arrival rates, the job shop can be maintained at a particular utilization level. For this particular mode, due to the its configuration (number of machines, operating times) the average shop utilization rate is equal to the average arrival rate. Due-dates of jobs are determined using the Total Work Content (TWK) method (Baker 1984). Operation due-dates are set in proportion to the operation processing times. We have selected 12 rules which are either extensively used (such as FIFO, EDD, SLACK) or which perform very well for tardiness based measures (see Table 5 for a list and definition of these rules). Table 1 summarizes the parameter settings used in the simulation model.

Table 1.: Simulation model parameters

\begin{tabular}{ll}
\hline Parameter & Value \\
\hline Number of machines & 4 \\
Number of completed jobs & 5,000 and 500,000 \\
Number of operations & $\mathrm{U}[2,6]$ \\
Warm-up period & 1,000 time units \\
Number of replications & 100 and 1 \\
Job routings & random with limited revisiting \\
Job release policy & Immediate \\
Release dates & $\operatorname{EXP}\left[\frac{1}{\eta}\right]$ \\
Operation processing times & $\operatorname{EXP}[1]$ \\
Tie-breaking rule & First in Queue \\
Due-date assignment method & TWK \\
\hline
\end{tabular}

Research investigations have focused primarily on the relative effectiveness of various priority dispatching rules in job shops. For due-date based objectives, the relative performance of these pdrs is affected by the quality of the due-date assignment methods (such as Total Work Content (TWK)) (Baker 1984), the due-date tightness $(\tau)$ and due-date variation $(\rho)$ (Cheng and Gupta 1989, Lejmi and Sabuncuoglu 2002). The TWK method 
is given as

$$
d_{j}=r_{j}+\omega \sum_{i=1}^{o_{j}} p_{j(i)},
$$

where $r_{j}$ is the release date of the $j^{\text {th }}$ job, $d_{j}$ is the due-date of the $j^{\text {th }}$ job, $\sum_{i=1}^{o_{j}} p_{j(i)}$ is the sum of processing times of all the operations of $j^{\text {th }}$ job and $\omega$ is a multiplier given as $\omega=U N I F[\tau-\rho, \tau+\rho] . \tau$ represents the due-date tightness whereas $\rho$ is the due-date variability. This allows job due-dates to be generated within a desired range of tightness and variability.

As in this study, we consider only due-date tightness as a varying condition for the due-dates, we have $\omega=\tau$, implying no due-date variation. The relative ranking of pdrs is affected by such varying conditions for some specified tardiness based measure. For example, tighter due-dates tend to produce larger values for $\bar{T}$ and $\% T$, while keeping other conditions unchanged (Carroll 1965).

There are $2 \times 3=6$ configurations to be simulated (see Table 2) for each pdr. Two levels of shop load are defined. A moderate shop load level, which corresponds to a utilization rate of $80 \%$ for the resources, and a high level of shop load, which corresponds to utilization rate of $90 \%$. For each load level, three different levels of due-date were established. Each given due-date tightness (e.g., tight, moderate or loose) is computed in a proportion depending on the utilization rate of the resources (Schultz 1989, Pierreval and Mebarki 1997). This means that the allowance for a job depends on the utilization rate of the resources and the desired due-date tightness. Thus, for the FIFO rule, with tight due-dates $60 \%$ of the jobs are found late in our model.

This job shop model is a hypothetical model but it presents some advantages: as it is a generic stochastic model, it is not dependant on a particular kind of industry. Moreover, it is also quite complex model since the routings are random (as well as the arrival rate and the processing times) and the average number of operations is equal to the number of machines.

Firstly, the performance measures for the set of pdrs were collected for 5,000 jobs over 100 replications with a warm-up period of 1,000 time units (corresponding approximately to 500 jobs) under given operating conditions. Then one long simulation for 500,000 jobs is carried out to obtain the distributions of tardiness values for these pdrs under the same operating conditions. The 12 rules were chosen either beacuse they exhibit very good performance for tardiness based performance (CRSI, COVERT, MOD, CEXSPT, ATC, MF) (Kemppainen 2005) or because they are standard pdrs largely used in industry and in the literature (FIFO, SI, SPT, EDD, SLACK, CR).

\section{Results and Discussion}

Fig. 1 presents the box-plots of $T_{\max }$ for the set of pdrs under different operating conditions for 100 replications. For each rule, there are 6 box-plots, each one corresponding to a particular operating condition.

From these box-plots, we can identify two sets of rules based on their behavior in regards with $T_{\max }$.

- FIFO, EDD, SLACK, CR noted as set 1 for which $T_{\max }$ is quite low with less degree of dispersion and there are very few outliers. 
Table 2.: Operating conditions tested.

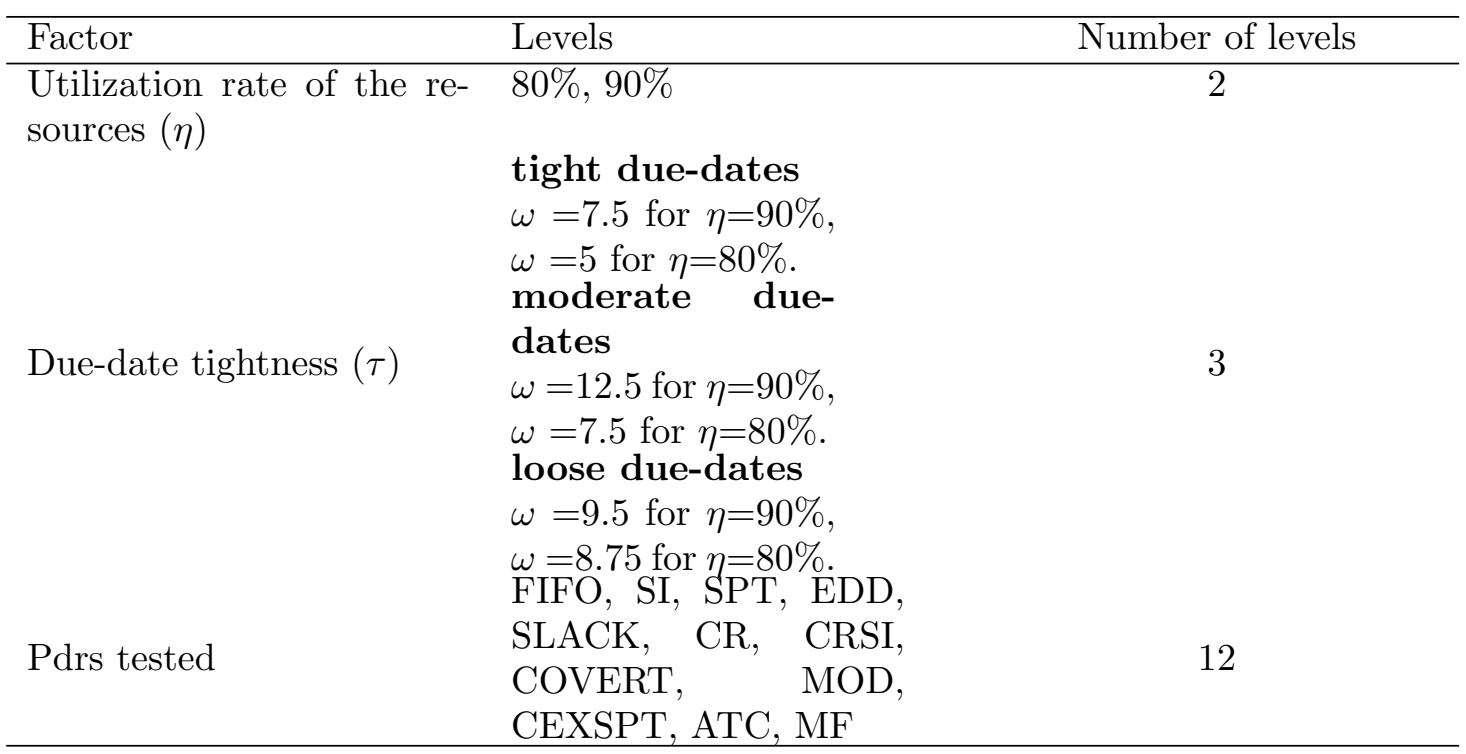

- SI, SPT, MOD, MF noted as set 2 for which there is a much higher degree of dispersion for $T_{\max }$ with larger number of extreme outliers.

For example, in the case of SI rule under high load and tight due-date conditions, inter-quartile range (IQR is the range within which the middle $50 \%$ of the ranked data is found) is 301.5 (=744.3-442.5) with two extreme outliers at 2369.3 and 2446.5. On the other hand, for SLACK rule under similar conditions, IQR is merely 19.8 with only one mild outlier at 97.6.

For the other rules (CR/SI, COVERT, CEXSPT, ATC) it can be observed that these rules change their behavior in regards with the varying conditions of load and due-date tightness. For tight due-date settings, the behavior of these rules compares to rules of set 1 . However, as the due-dates get looser, these rules tend to behave like rules of set 2. This behavior is inherent to the structure of these rules (and hence generally referred as trade-off heuristics). For example CR/SI does not take into account the amount of relative lateness and it has two different behaviors for late jobs and non-late jobs.

Under higher loading conditions, the performance of the pdrs deteriorate however, there is no significant impact on the relative ranking of the pdrs.

The corresponding distribution plots of the tardiness values, obtained through a single long simulation run, for these two sets of rules are shown in Fig. 2 and Fig. 3 . These distribution plots for a single long simulation run clearly show two different kinds of behaviors for rules of set 1 and rules of set 2 . Our measures show that for rules of set 1 , the tardiness values are quite evenly distributed in contrast to the rules of set 2 where the concentration of tardiness is only on a few jobs, that are very tardy, justifying the high value of maximum tardiness for rules of set 2 . This is a typical behavior that is measured by $T_{r m s}$, as mentioned earlier. In order to check if the two sets of rules previously identified in regards with the $T_{\max }$, can also be discriminated by using the $T_{r m s}$, it is decided to measure the relative performance of these benchmark pdrs in regards with the $T_{r m s}$. 
High Load
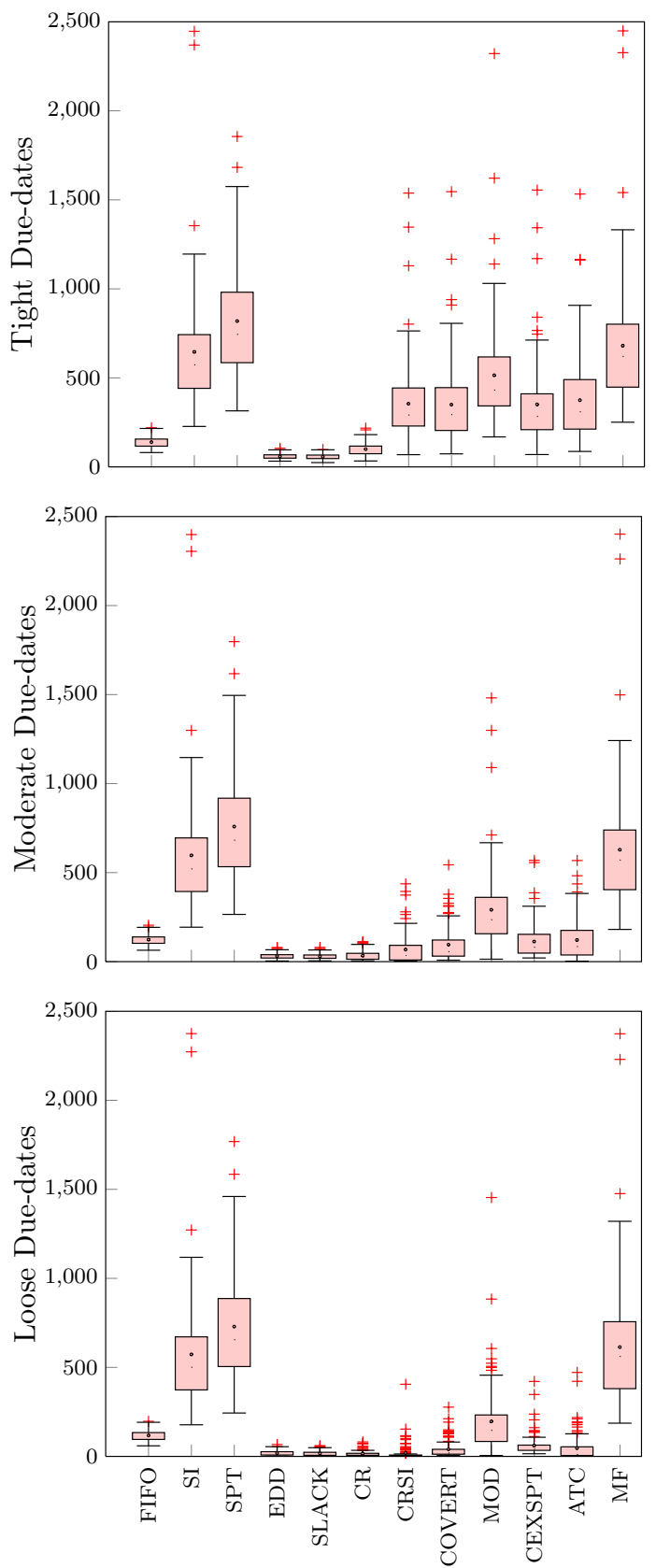

Moderate Load
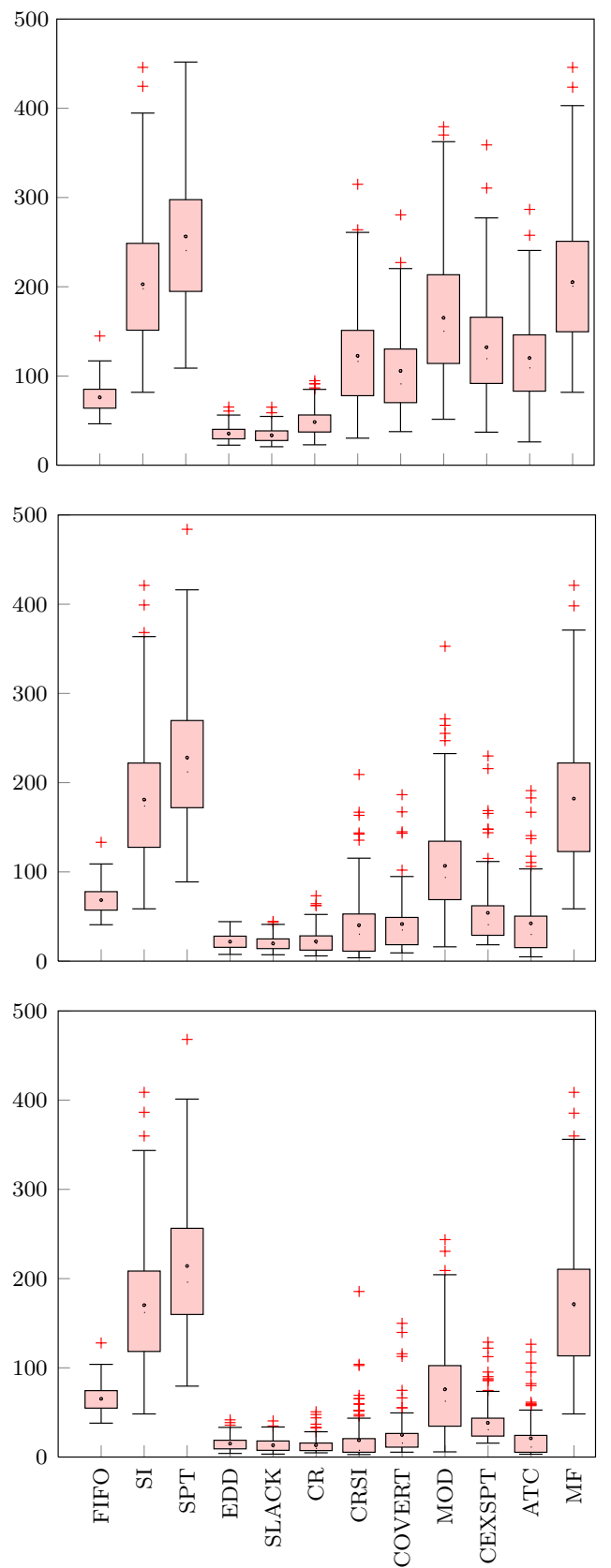

Figure 1.: Box-plots of $T_{\max }$ with corresponding $(\tau, \eta)$

Fig. 4 present a comparison of $T_{\max }$ and $T_{r m s}$ under different operating conditions within the two sets of rules previously identified and other rules. Rules of set 1 perform significantly well for $T_{\max }$, with SLACK rule as always the best performing rule. For rules of set 1, EDD and SLACK exhibit quite a similar behavior, however for FIFO, $T_{\max }$ and $T_{r m s}$ are not as strongly correlated as the other two pdrs of this set. Probably, 


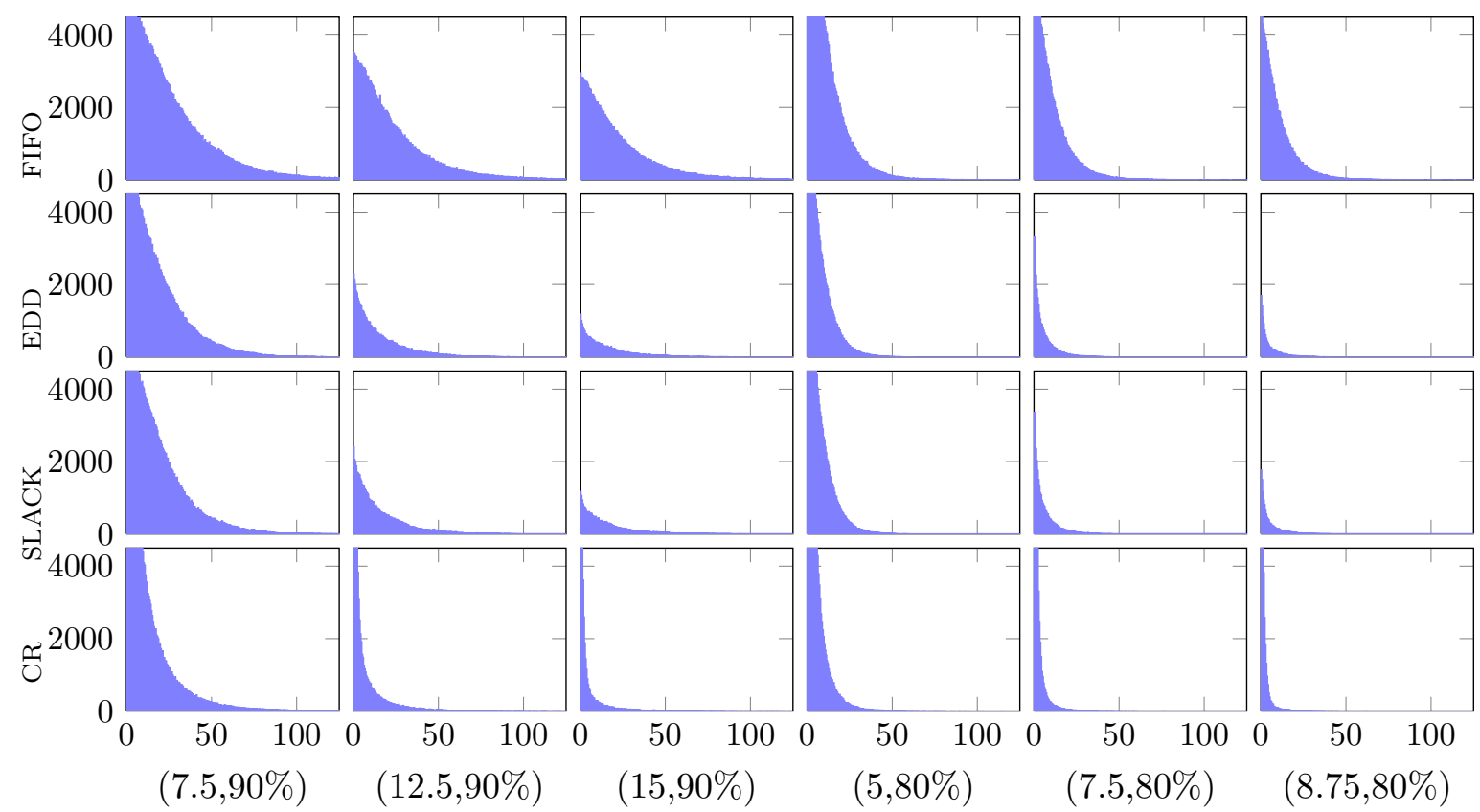

Figure 2.: Tardiness distribution for rules of set 1 with corresponding $(\tau, \eta)$

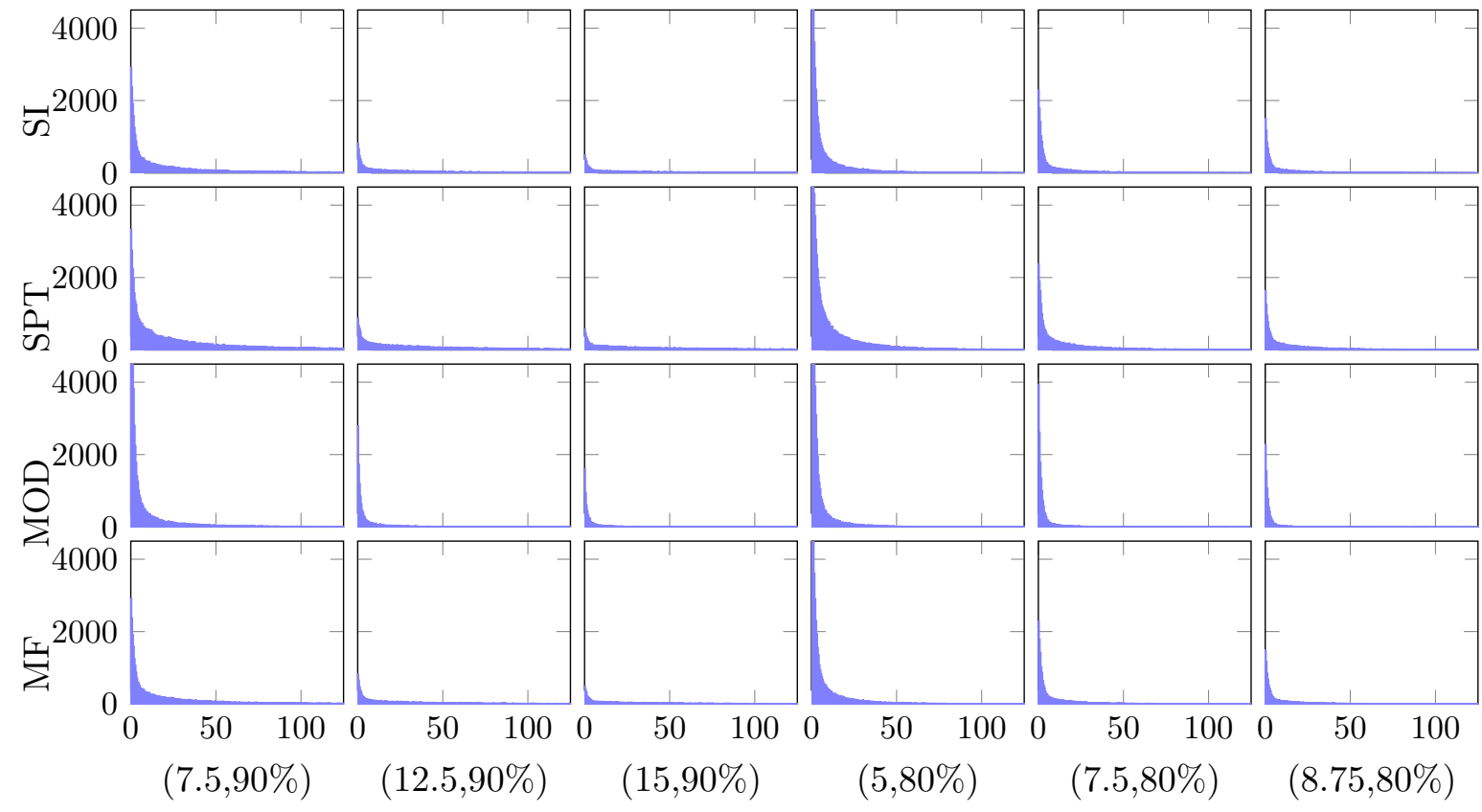

Figure 3.: Tardiness distribution for rules of set 2 with corresponding $(\tau, \eta)$

this is due to the fact that, in FIFO there is neither the involvement of due-date factor 
nor the processing times resulting in higher values for tardiness (as compared to other two rules of set 1$)$.

Table 3.: Confidence Interval for Correlation between $T_{\max }$ and $T_{r m s}$

\begin{tabular}{|c|c|c|c|c|c|c|}
\hline$(\omega, \eta)$ & $(7.5,90 \%)$ & $(12.5,90 \%)$ & $(15,90 \%)$ & $(5,80 \%)$ & $(7.5,80 \%)$ & $(8.75,80 \%)$ \\
\hline \multirow{2}{*}{ FIFO } & 0.85529 & 0.85963 & 0.85604 & 0.74663 & 0.75248 & 0.75248 \\
\hline & {$[0.7926,0.89986]$} & {$[0.79261,0.90482]$} & {$[0.78931,0.90291]$} & {$[0.64096,0.84518]$} & {$[0.64177,0.86386]$} & {$[0.64177,0.86386$} \\
\hline \multirow{2}{*}{ SI } & 0.89076 & 0.90969 & 0.91798 & 0.82116 & 0.85957 & 0.85957 \\
\hline & {$[0.81299,0.94655]$} & {$[0.84568,0.95843]$} & {$[0.85023,0.96146]$} & {$[0.73265,0.89054]$} & {$[0.7919,0.91477]$} & {$[0.7919,0.91477]$} \\
\hline \multirow{2}{*}{ SPT } & 0.87495 & 0.88438 & 0.88884 & 0.77426 & 0.80669 & 0.80669 \\
\hline & {$[0.81709,0.91214]$} & {$[0.83268,0.91846]$} & {$[0.84133,0.92192]$} & {$[0.68927,0.83444]$} & {$[0.73219,0.86285]$} & {$[0.73219,0.86285]$} \\
\hline \multirow{2}{*}{ EDD } & 0.87681 & 0.91346 & 0.89915 & 0.82829 & 0.88431 & 0.88431 \\
\hline & {$[0.80323,0.92411]$} & {$[0.87314,0.93581]$} & {$[0.85284,0.92789]$} & {$[0.73672,0.89456]$} & {$[0.84067,0.91658]$} & {$[0.84067,0.91658]$} \\
\hline \multirow{2}{*}{ SLACK } & 0.87442 & 0.92353 & 0.9143 & 0.82894 & 0.92511 & 0.92511 \\
\hline & {$[0.80542,0.91772]$} & {$[0.89158,0.9479]$} & {$[0.88377,0.94044]$} & {$[0.74006,0.89895]$} & {$[0.89416,0.94954]$} & {$[0.89416,0.94954]$} \\
\hline \multirow{2}{*}{$\mathrm{CR}$} & 0.88691 & 0.94371 & 0.94926 & 0.80228 & 0.92711 & 0.92711 \\
\hline & {$[0.81835,0.92796]$} & {$[0.91115,0.96202]$} & {$[0.91001,0.96929]$} & {$[0.71576,0.86626]$} & {$[0.88473,0.959]$} & {$[0.88473,0.959]$} \\
\hline \multirow{2}{*}{$\mathrm{CR} / \mathrm{SI}$} & 0.92886 & 0.95837 & 0.98692 & 0.87255 & 0.95654 & 0.95654 \\
\hline & {$[0.88921,0.96166]$} & {$[0.91968,0.97568]$} & {$[0.96101,0.99877]$} & {$[0.80903,0.91708]$} & {$[0.93018,0.97198]$} & {$[0.93018,0.97198]$} \\
\hline \multirow{2}{*}{ CoverT } & 0.91365 & 0.9529 & 0.93416 & 0.85643 & 0.90983 & 0.90983 \\
\hline & {$[0.8615,0.94638]$} & {$[0.92114,0.97366]$} & {$[0.88398,0.97113]$} & {$[0.77751,0.91454]$} & {$[0.83947,0.95133]$} & {$[0.83947,0.95133]$} \\
\hline \multirow{2}{*}{ MOD } & 0.88924 & 0.93424 & 0.95677 & 0.86473 & 0.89796 & 0.89796 \\
\hline & {$[0.83239,0.93016]$} & {$[0.89132,0.96964]$} & {$[0.93001,0.96886]$} & {$[0.80519,0.90954]$} & {$[0.85873,0.92713]$} & {$[0.85873,0.92713]$} \\
\hline \multirow{2}{*}{ CEXSPT } & 0.92407 & 0.95033 & 0.95927 & 0.83688 & 0.90615 & 0.90615 \\
\hline & {$[0.88205,0.96247]$} & {$[0.90848,0.97623]$} & {$[0.91218,0.98616]$} & {$[0.7736,0.88755]$} & {$[0.8533,0.93747]$} & {$[0.8533,0.93747]$} \\
\hline \multirow{2}{*}{ ATC } & 0.92855 & 0.95065 & 0.9859 & 0.8715 & 0.94496 & 0.94496 \\
\hline & {$[0.89352,0.95382]$} & {$[0.91384,0.96863]$} & {$[0.9693,0.99507]$} & {$[0.81272,0.91484]$} & {$[0.91124,0.96844]$} & {$[0.91124,0.96844]$} \\
\hline \multirow{2}{*}{$\mathrm{MF}$} & 0.9091 & 0.92447 & 0.925 & 0.80034 & 0.83919 & 0.83919 \\
\hline & {$[0.84872,0.95256]$} & {$[0.86551,0.96275]$} & {$[0.86443,0.96358]$} & {$[0.70001,0.87661]$} & {$[0.75322,0.90042]$} & {$[0.75322,0.90042]$} \\
\hline
\end{tabular}

For stochastic models, it is required to construct a confidence interval for a parameter estimate in statistical inferences. Bootstrapping procedure is used to obtain $95 \%$ confidence intervals for the correlation coefficients between $T_{\max }$ and $T_{r m s}$, which are presented in Table 3. For SI rule, for example, under high load and tight due-date conditions (i.e. $(7.5,90 \%)$ ), the confidence limits for the correlation coefficient is 0.813 (lower limit) and 0.947 (upper limit). Table 3 presents strong quantitative evidence that $T_{\max }$ and $T_{r m s}$ are positively correlated. Moreover, this evidence does not require any strong 

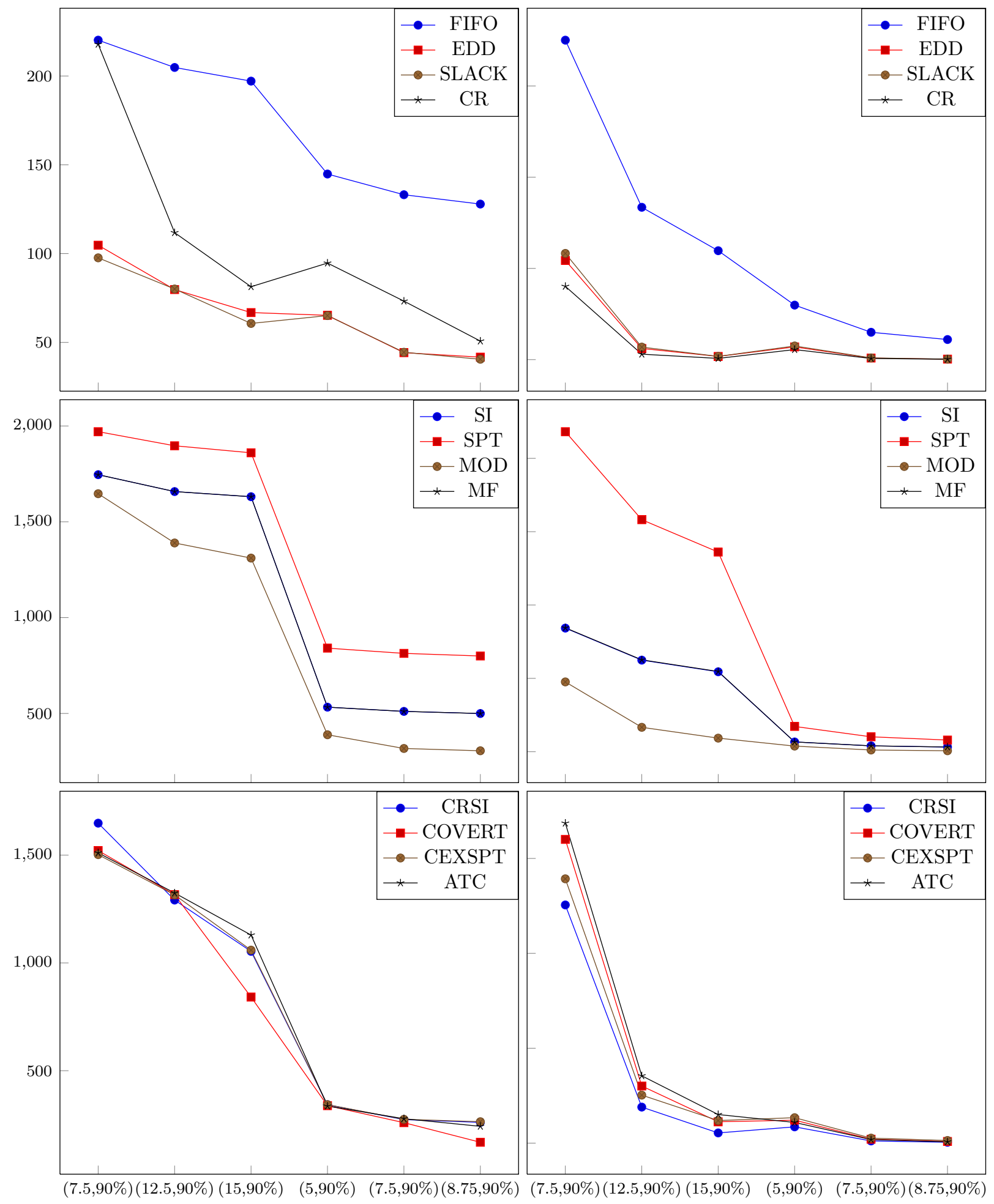
(a) $T_{\max }$
(b) $T_{r m s}$

Figure 4.: Comparison of $T_{\max }$ and $T_{r m s}$ for rules 
assumptions about the probability distribution of the correlation coefficient. It is also observed that this correlation is stronger under High load and loose due-date settings for all the rules.

As a matter of fact, the root-mean-square tardiness $\left(T_{r m s}\right)$ is a very interesting measure. It has three advantages. First, and this is its usual use, it permits to differentiate a system with a little number of tardy jobs having higher values for tardiness from a system with a lot of tardy jobs having low tardiness. Second, we have demonstrated that it can also be used along with mean tardiness to compute an estimate of the variance of the tardiness. And third, we have also tried to show that it gives an indication of the relative performance of the rules for $T_{\max }$ as $T_{r m s}$ is found to be strongly correlated with the maximum tardiness. 


\section{Conclusions}

In this article, we have discussed tardiness based performance measures using a set of pdrs that are extensively used in the literature on scheduling. Based on the behavior of pdrs in regards with maximum tardiness, two sets of rules have been identified; FIFO, EDD, SLACK and CR from one side and SI, SPT, MOD and MF on the other side. The behavior for the rules of the first set is to exhibit a large number of tardy jobs with low tardiness, which is exactly opposite to that for the rules of the second set. There are some rules (CRSI, COVERT, CEXSPT, ATC) exhibiting these two behaviors under different conditions. For tight due-date setting, these rules behave more like the rules of set 1 while under loose due-date setting, their behavior tends towards rules of set 2 . This kind of discrimination is typically found through the $T_{r m s}$.

We found results that show a strong correlation between the $T_{\max }$ and the $T_{r m s}$ in our experiments. This correlation is found to be relatively stronger in congested shops. The worst case performance in regards with tardiness of a particular priority dispatching rule may therefore be predicted by evaluation of $T_{r m s}$ which can be used along with the mean tardiness to compute the variance of the tardiness. So, the $T_{r m s}$ can be used to rank the priority dispatching rules in regards with the $T_{\max }$ and the width of the tardiness. For a company which wants to put a limit on the tardiness, this study shows the effectiveness of the pdrs described in set 1 (i.e., FIFO, EDD, SLACK and CR). These rules are quite easy to implement and are already frequently used in industrial manufacturing systems. This study also shows that depending on the operating conditions (i.e., tight due dates or not, moderate shop load or not), it can be interesting to use more sophisticated rules such as CRSI, COVERT, CEXSPT or ATC in order to put a limit on the tardiness. In all cases, it is valuable to simulate the manufacturing system using priority dispatching rules to evaluate the maximum tardiness along with the root-mean square tardiness in order to put a limit on the tardiness.

The results presented in this study are based on a simulation model that has become a benchmark for job shop configuration. The set of priority dispatching rules used in this study are not tied to some specific scheduling environment. Thus this can be safely assumed that results are not specific to this particular model or limited to a particular set of priority dispatching rules. However an extended study on the behavior of these priority dispatching rules in regards with other parameters (e.g. due-date variability, due-date methods and anticipated waiting times) would give more insights to the inter-relation of these tardiness based measures. This would result in a more general classification scheme of priority dispatching rules for the tardiness based measures. It would also be an interesting research topic to study the effects of different routing schemes on these results.

\section{References}

Baker, K., 1984. Sequencing rules and due-date assignments in a job shop. Management Science, 30 (9), 1093-1104.

Baker, K.R. and Bertrand, J.W.M., 1982. A dynamic priority rule for scheduling against due-dates. Journal of Operations Management, 3 (1), 37 - 42.

Baker, K., 1974. Introduction to sequencing and scheduling. Wiley New York.

Baker, K. and Kanet, J., 1983. Job shop scheduling with modified due dates. Journal of Operations Management, 4 (1), 11-22. 
Carroll, D., 1965. Heuristic sequencing of jobs with single and multiple components. Thesis (PhD). Sloan School of Management, MIT.

Chang, P. and Su, L., 2001. Scheduling $\mathrm{n}$ jobs on one machine to minimize the maximum lateness with a minimum number of tardy jobs. Computers $\&$ industrial engineering, 40 (4), 349-360.

Cheng, T. and Gupta, M., 1989. Survey of scheduling research involving due date determination decisions. European Journal of Operational Research, 38 (2), 156-166.

Dominic, P.D.D., Kaliyamoorthy, S., and Kumar, M.S., 2004. Efficient dispatching rules for dynamic job shop scheduling. The International Journal of Advanced Manufacturing Technology, 24, 70-75 10.1007/s00170-002-1534-5.

Eilon, S. and Cotterill, D., 1968. A modified SI rule in job shop scheduling. International Journal of Production Research, 7 (2), 135-145.

French, S., 1982. Sequencing and Scheduling: An Introduction to the Mathematics of the Job Shop. Ellis Horwood Ltd.

Kemppainen, K., 2005. Priority scheduling revisited - dominant rules, protocols and integerated order management. Thesis (PhD). Helsinki School of Economics.

Laslo, Z., 2010. Project portfolio management: An integrated method for resource planning and scheduling to minimize planning/scheduling-dependent expenses. International Journal of Project Management, 28, 609-618.

Laslo, Z., Golenko-Ginzburg, D., and Keren, B., 2008. Optimal booking of machines in a virtual job-shop with stochastic processing times to minimize total machine rental and job tardiness costs. International Journal of Production Economics, 111, 812-821.

Lejmi, T. and Sabuncuoglu, I., 2002. Effect of load, processing time and due date variation on the effectiveness of scheduling rules. International Journal of Production Research, 40 (4), 945 - 974.

Mainieri, G. and Ronconi, D., 2010. New heuristics for total tardiness minimization in a flexible flowshop. Optimization Letters, 1-20 10.1007/s11590-012-0448-x.

Malve, S. and Uzsoy, R., 2007. A genetic algorithm for minimizing maximum lateness on parallel identical batch processing machines with dynamic job arrivals and incompatible job families. Computers \& Operations Research, 34 (10), 3016-3028.

Mattfeld, D.C. and Bierwirth, C., 2004. An efficient genetic algorithm for job shop scheduling with tardiness objectives. European Journal of Operational Research, 155 (3), $616-630$.

Moser, M. and Engell, S., 1992. A survey of priority rules for FMS scheduling and their performance for the benchmark problem. In: Proceeding of the 31st Conference on Decision and Control, Vol. 1 Arizona: IEEE, 392-397.

Panwalker, S. and Iskander, W., 1977. A survey of dispatching rules. Operations Research, $25,45-61$.

Pierreval, H. and Mebarki, N., 1997. Dynamic selection of dispatching rules for manufacturing system scheduling. International Journal of Production Research, 35 (6), $1575-1591$.

Pinedo, M.L., 2008. Scheduling: Theory, Algorithms, and Systems. Springer.

Raman, N., Talbot, F.B., and Rachamadugu, R.V., 1989. Due date based scheduling in a general flexible manufacturing system. Journal of Operations Management, 8 (2), $115-132$.

Russell, R., Dar-El, E., and Taylor, B., 1987. A comparative analysis of the COVERT job sequencing rule using various shop performance measures. International Journal of Production Research, 25 (10), 1523-1540.

Schultz, C.R., 1989. An expediting heuristic for the shortest processing time dispatching 
rule. International Journal of Production Research, 27, 31-41.

\section{Appendix A}

Table 4.: Glossary of Notations

\begin{tabular}{ll}
\hline Symbol & Definition \\
\hline$i$ & Index for an operation \\
$j$ & Index for a job \\
$k$ & Index for a machine \\
$n$ & Number of jobs \\
$r_{j}$ & Release date of $j^{\text {th }}$ job \\
$d_{j}$ & Due-date of $j^{\text {th job }}$ \\
$p_{j}$ & Sum of processing times of all operations of $j^{\text {th }}$ job \\
$C_{j}$ & Completion time of last operation of $j^{\text {th }}$ job \\
$T_{j}$ & Tardiness of $j^{\text {th } \text { job }}$ \\
$W_{j}$ & Expected waiting time of $j^{\text {th }}$ job \\
$\lambda$ & Arrival rate of jobs \\
$\mu$ & Service rate of jobs \\
$\eta$ & Shop utlization level \\
$\rho$ & Due-date variability factor \\
$\tau$ & Due-date tightness factor \\
$\omega$ & Due-date allowance multiplier \\
$(x)^{+}$ & $=\max (x, 0)$ \\
\hline
\end{tabular}


Table 5.: Definitions of Benchmark Priority Dispatching Rules

\begin{tabular}{|c|c|c|c|}
\hline Rule & Definition & Rank & Priority Index \\
\hline$\overline{\text { FIFO }}$ & First In First Out & $\min$ & $C_{j(i-1)}$ \\
\hline SI & $\begin{array}{l}\text { Shortest Imminent } \\
\text { processing time }\end{array}$ & $\min$ & $p_{j(i)}$ \\
\hline SPT & $\begin{array}{l}\text { Shortest Processing } \\
\text { Time }\end{array}$ & $\min$ & $p_{j}$ \\
\hline EDD & Earliest Due-Date & $\min$ & $d_{j}$ \\
\hline SLACK & Slack & $\min$ & $d_{j}-t-\sum_{i=l}^{o_{j}} p_{j(i)}$ \\
\hline $\mathrm{CR}$ & Critical Ratio & $\min$ & $\frac{d_{j}-t}{\sum_{i=l}^{o_{j}} p_{j(i)}}$ \\
\hline CRSI & $\begin{array}{l}\text { Critical Ratio/Shortest } \\
\text { Imminent }\end{array}$ & $\min$ & $p_{j k} \times \max \left(\frac{d_{j}-t}{\sum_{i=l}^{o_{j}} p_{j(i)}}, 1\right)$ \\
\hline MOD & $\begin{array}{l}\text { Modified Operation } \\
\text { Due-date }\end{array}$ & $\min$ & $\max \left(d_{j k}, t+p_{j k}\right)$ \\
\hline COVERT & Cost Over Time & $\max$ & $\frac{1}{p_{j(i)}}\left(1-\frac{\left(d_{j}-t-\sum_{i=l}^{o_{j}} p_{j(i)}\right)^{+}}{h_{1} \sum_{l=i}^{o_{j}} W_{j(l)}}\right)^{+}$ \\
\hline ATC & $\begin{array}{l}\text { Apparent Tardiness } \\
\text { Cost }\end{array}$ & $\max$ & $\frac{1}{p_{j(i)}} \exp \left(-\frac{\left(d_{j}-t-p_{j(i)}-h_{2} \sum_{i=l+1}^{o_{j}} p_{j(i)}\right)}{h_{3} \sum_{i=l}^{o_{j}} p_{j(i)}}\right)^{+}$ \\
\hline MF & Multi-Factor & $\max$ & $\frac{1}{p_{j(i)}}\left(W_{j(i)}-\left(d_{j}-t-\sum_{i=l}^{o_{j}} p_{j(i)}\right)\right)$ \\
\hline CEXSPT & $\begin{array}{l}\text { Conditionally } \\
\text { Expedited SPT }\end{array}$ & - & $\begin{array}{l}W_{j(q)}=\sum_{i=k}^{n(\neq k)} \sum_{j=1}^{q} p_{j(i)}-\sum_{j=1}^{q-1} p_{j(i)} \text {. } \\
\text { Partition into three queues, late queue, opera- } \\
\text { tionally late queue and ahead-of-schedule queue, } \\
\text { with SI as selection criterion within queues. Shift- } \\
\text { ing of job to other queues is not allowed. }\end{array}$ \\
\hline
\end{tabular}

